\title{
OPEN Comparative analysis of nuclear, chloroplast, and mitochondrial genomes of watermelon and melon provides evidence of gene transfer
}

\author{
Haonan Cuil, ${ }^{1,2}$ Zhuo Ding ${ }^{1,2}$, Qianglong Zhu ${ }^{3}$, Yue Wu ${ }^{1,2}$, Boyan Qiu' ${ }^{1,2}$ \& Peng Gao ${ }^{1,2}$
}

During plant evolution, there is genetic communication between organelle and nuclear genomes. A comparative analysis was performed on the organelle and nuclear genomes of the watermelon and melon. In the watermelon, chloroplast-derived sequences accounted for $7.6 \%$ of the total length of the mitochondrial genome. In the melon, chloroplast-derived sequences accounted for approximately $\mathbf{2 . 7 3 \%}$ of the total mitochondrial genome. In watermelon and melon, the chloroplast-derived smallfragment sequences are either a subset of large-fragment sequences or appeared multiple times in the mitochondrial genome, indicating that these fragments may have undergone multiple independent migration integrations or emerged in the mitochondrial genome after migration, replication, and reorganization. There was no evidence of migration from the mitochondria to chloroplast genome. A sequence with a total length of about $73 \mathrm{~kb}(47 \%)$ in the watermelon chloroplast genome was homologous to a sequence of about $313 \mathrm{~kb}$ in the nuclear genome. About $33 \%$ of sequences in the watermelon mitochondrial genome was homologous with a $260 \mathrm{~kb}$ sequence in the nuclear genome. A sequence with a total length of about $38 \mathrm{~kb}(25 \%)$ in the melon chloroplast genome was homologous with 461 sequences in the nuclear genome, with a total length of about $301 \mathrm{~kb}$. A $3.4 \mathrm{Mb}$ sequence in the nuclear genome was homologous with a melon mitochondrial sequence. These results indicate that, during the evolution of watermelon and melon, a large amount of genetic material was exchanged between the nuclear genome and the two organelle genomes in the cytoplasm.

Watermelon (Citrullus lanatus [Thunb.] Matsum. Et Nakai) originated in Africa ${ }^{1}$, and Melon (Cucumis melo L.) originated in Africa ${ }^{2}$ or Asia ${ }^{3}$. Both are annual dicotyledonous plants of the genus Cucurbitaceae. Studies have shown that the chloroplast and mitochondrial genomes of watermelon and melon are related to important physiological activities, such as photosynthesis, respiration, cold tolerance, and sex differentiation ${ }^{4,5}$, and they are also useful for germplasm resource identification and phylogenetic research ${ }^{3,6}$.

Chloroplasts have bilayer membrane structures and are organelles particular to plant cells. The chloroplast genome of higher plants is generally between 115 and $165 \mathrm{~kb}$, and the structure is highly conserved. The chloroplast genome of tobacco was the first to be sequenced in higher plants ${ }^{7}$; its genome comprises a single-loop double-stranded DNA molecule with a typical structure of four segments, each of which has a large single-copy (LSC) region, a small single-copy (SSC) region, and two inverted repeat (IR) regions (IRa and IRb) ${ }^{8}$. The LSC region in the chloroplast genomes of most plants is between 81 and $90 \mathrm{~kb}$, the SSC region is between 18 and $20 \mathrm{~kb}$, and the maximum variation in the length of the IR region is between 5 and $76 \mathrm{~kb}^{9}$. The LSC and SSC regions are separated by the two IR sequences. During the evolution of angiosperms, the order of these four parts of the chloroplast genome have remained almost unchanged.

Mitochondria are found in most cells and, like chloroplasts, are surrounded by a membrane bilayer. Compared with the smaller, conserved chloroplast genome, the plant mitochondrial genome has more complex structural features, usually comprised of a single double-stranded circular DNA molecule, such as in Arabidopsis ${ }^{10}$, watermelon, and zucchini ${ }^{11}$. Some mitochondrial genomes also exist as linear DNA molecules, such as that of rice ${ }^{12}$. In addition, multiple circular mitochondrial genomes have been found in a wide range of plants, such as wheat ${ }^{13}$,

${ }^{1}$ College of Horticulture and Landscape Architecture, Northeast Agricultural University, Harbin 150030, Heilongjiang, People's Republic of China. ${ }^{2}$ Key Laboratory of Biology and Genetic Improvement of Horticulture Crops (Northeast Region), Ministry of Agriculture, Harbin 150030, Heilongjiang, People's Republic of China. ${ }^{3}$ Department of Horticulture, College of Agronomy, Jiangxi Agricultural University, Nanchang, People's Republic of China. ${ }^{\varpi}$ email: gaopeng_neau@163.com 


\begin{tabular}{|l|l|l|}
\hline & Mitochondrial genomes & Chloroplast genomes \\
\hline Watermelon & NC_014043 & NC_032008 \\
\hline \multirow{3}{*}{ Melon } & MG947207 & \multirow{3}{*}{ NC_015983 } \\
\cline { 2 - 2 } & MG947208 & \\
\cline { 2 - 3 } & MG947209 & \\
\hline
\end{tabular}

Table 1. GeneBank numbers for watermelon and melon organelle genomes.

rape $^{14}$, and cucumber ${ }^{15}$. Although the number of genes encoded by the mitochondrial genome varies from plant to plant, the types and sequences of the genes are very conserved. In fact, the evolution rate is the slowest and most conservative of the three sets of plant genomes, and the position and arrangement are quite different ${ }^{16}$.

In theory, there are six types of sequence migration or endogenous gene transfer in cells. That is, when a nuclear sequence is endogenously transferred into the mitochondria or chloroplasts, mitochondrial sequences are endogenously transferred to the nucleus or chloroplasts, and chloroplast sequences are endogenously transferred to the nucleus or mitochondria ${ }^{17-24}$. In general, plastid genomes are thought to be highly conservative and do not integrate much foreign genetic material ${ }^{25}$. Although, the mitochondrial genome of seed plants is thought to integrate sequences from chloroplasts, the nucleus ${ }^{26-28}$, and other mitochondria ${ }^{29}$.

The intergenic region accounts for a large proportion of the plant mitochondrial genome and is poorly conserved. It mainly includes foreign migrating fragments and chimeric sequences composed of repetitive segments mediated through recombination. The sequences of exogenous migrating fragments are mainly obtained from the chloroplast and nuclear genomes via horizontal gene transfer, but they may also be transferred from bacterial genomes, such as was found in the mitochondrial genome of cucumber ${ }^{15}$. Repetitive sequences are the most common and important sequences in the mitochondrial intergenic region. They are related to the mutation and recombination of the mitochondrial genome and play an important role in the evolution of plant mitochondrial genomes.

In this study, we compared sequence homologies between chloroplast, mitochondria, and nuclear genomes to estimate the amount of genetic material exchanged between the organelles and nuclei in watermelon and melon. In addition, we aimed to provide basic data for future related research into watermelon and melon nuclear-cytoplasm interactions.

\section{Materials and methods}

DNA isolation, genome sequencing and assembly of Watermelon and Melon. The watermelon cultivars W1-1 and melon cultivars MR-1 were planted in a plastic greenhouse at the Horticultural Station of the Northeast Agricultural University, Harbin, China $\left(44^{\circ} 04^{\prime} \mathrm{N}, \mathrm{E} 125^{\circ} 42^{\prime}\right)$. And the individuals were using standard horticultural procedures (irrigation, hand-weeding, and pathogen prevention and control) typical of the climatic conditions in Harbin.

Young leaves without any damage of W1-1 and MR-1 were collected and quickly transferred into storage at $-80^{\circ} \mathrm{C}$ until DNA extraction. Total DNA was extracted using the modified cetyl trimethyl ammonium bromide $\operatorname{method}^{30}$. The ultrasonically fragmented DNA samples were purified, distally repaired, ligated sequencing joint, then were chosen by $1 \%$ agarose gel electrophoresis to obtain a target insert size of $500-600$ bp and purified for further analysis. PCR amplification was performed to construct a paired-read sequencing library. The DNA libraries were sequenced on an Illumina HiSeqTM 2500 platform (Biomarker, Beijing, China) to generate 125 base paired-end reads. The Re-Sequencing data of W1-1 and MR-1 were deposited in GenBank (https://www. ncbi.nlm.nih.gov/) under BioProject ID PRJNA682698.

Alignment of paired-end reads to the reference sequence. We downloaded chloroplast and mitochondrial genomic data for the watermelon and melon from the NCBI database (Table 1), and we downloaded watermelon $(97,103)$ genome $2^{31}$ and melon (DHL92) genome 3.5.1 $1^{32}$ from the Cucurbitaceae genome website (http://cucurbitgenomics.org).

To obtain clean, high quality reads, the raw reads of all samples were filtered according to strict parameters as described previously ${ }^{33}$. After the reads were checked for quality, the cleaned reads of W1-1 and MR-1 were aligned to the reference genome using built-in BWA aligner (version 0.6.1-r104) ${ }^{34}$. Sequences with sequencing depth greater than 30 were selected as credible watermelon and melon nuclear genomes, chloroplast genomes and mitochondrial genomes for subsequent analysis.

Comparative analysis of organelle and nuclear genomes. Genomic-scale sequence comparisons were carried out with LASTv7.1.4 $4^{35}$ to map regions sharing similar sequences within a genome and to identify the sequences shared between chloroplast and mitochondrial genomes. The software HS-BLASTN v0.0.5 $5^{36}$ and Tbtools v1.064 $4^{37}$ were used to analyze the sequence comparisons between the organelle and nuclear genomes, with all software parameters set to default.

Statistical analysis and graphics. All of the statistical analyses were performed using R v3.3.3 $3^{38}$, and the figures were generated using CIRCUS ${ }^{39}$ and ggplot $2^{40}$ in R. 


\begin{tabular}{|l|l|l|}
\hline Features & Chloroplast & Mitochondrion \\
\hline Genome size (bp) & 156,906 & 379,236 \\
\hline Chromosome number & 1 & 1 \\
\hline GC (\%) & 37.18 & 45.08 \\
\hline Gene number & 130 & 63 \\
\hline Protein-coding gene number & 86 & 39 \\
\hline Protein-coding sequence (bp) & $78,468(50.01 \%)$ & $32,553(8.58 \%)$ \\
\hline Genes with introns (cis/trans) & $18 / 2$ & $11 / 3$ \\
\hline Intron no. (cis/trans) & $24 / 2$ & $20 / 4$ \\
\hline Cis-splicing intron (bp) & $19,599(12.59 \%)$ & $32,735(8.63 \%)$ \\
\hline tRNA number & $37(30)$ & $18(15)$ \\
\hline tRNA sequence (bp) & $2807(1.80 \%)$ & $1454(0.38 \%)$ \\
\hline rRNA number & $8(4)$ & $3(3)$ \\
\hline rRNA sequence (bp) & $9394(5.98 \%)$ & $5145(1.35 \%)$ \\
\hline
\end{tabular}

Table 2. The basic features of chloroplast and mitochondrial genomes in C. lanatus. The count excluding the gene duplicates. The note is applicable only to the numbers mentioned in brackets for features tRNA number and rRNA number.

\begin{abstract}
Results
Re-sequencing and assembly of nuclear genomic and organelle genomes in watermelon and melon. A total of 101,667,347 clean reads were obtained from resequencing of watermelon cultivated material W1-1, totaling 15,250,102,050 bp. And 14,945,100,009 bp were spliced to the nuclear genome, 13,894,024 bp to the mitochondrial genome, and $5,434,804 \mathrm{bp}$ to the chloroplast genome by comparison with the reference genome, with an average sequencing depth of 34 . A total of 75,866,316 clean reads were obtained from resequencing of melon cultivated material MR-1, totaling 11,379,947,400 bp. And 10,355,752,134 bp were spliced to the nuclear genome, $91,285,780 \mathrm{bp}$ to the mitochondrial genome, and 5,680,510 bp to the chloroplast genome by comparison with the reference genome, with an average sequencing depth of 30 . Sequences with sequencing depth greater than 30 were selected as credible watermelon and melon nuclear genomes, chloroplast genomes and mitochondrial genomes for subsequent analysis.
\end{abstract}

Comparison of chloroplast and mitochondrial genome characteristics of watermelon. Although the watermelon mitochondrial genome is about 2.4 times the size of the chloroplast genome (Table 2), the number of protein-coding genes was found to be only about one-half that of the chloroplast genome. The proteincoding genes of the chloroplast genome accounted for about $50 \%$ of the total genome length, while the protein-coding genes of the mitochondrial genome only comprised $8.58 \%$ of the genome. The 26 introns of the watermelon chloroplast genome were distributed among 18 genes, with a single gene having no more than two introns. The 24 introns of the watermelon mitochondria genome were distributed among 11 genes, and some genes, such as the nad gene family, nad2, nad5, and nad7, had up to four introns. The chloroplast genome of watermelon encodes a set of tRNA genes that it can use to synthesize its own proteins, whereas the mitochondrial genome lacks the tRNA to recognize some amino acids and needs to import tRNA from the cytoplasm for protein synthesis.

Sequence migration between chloroplast and mitochondrial genomes of watermelon. This study found that, in the mitochondrial genome of the watermelon, there were 34 chloroplast-derived sequences with a length of more than $50 \mathrm{bp}$, a similarity of no less than $80 \%$ (Fig. 1; Table 3), and a total length of 28,703 bp, accounting for about $7.6 \%$ of the mitochondria genome. The homologous sequences in the mitochondrial genome of the watermelon made up $18.3 \%$ of the total length of the chloroplast genome, in which there were 25 sequence fragments with a length of more than $100 \mathrm{bp}$ (Supplementary Table S1). Among these fragments, 12 fragments were $100-500 \mathrm{bp}$ in length, 2 fragments were $500-1000 \mathrm{bp}$, and 11 fragments were longer than $1 \mathrm{~kb}$. With regards to distribution, 16 were from the chloroplast genomic LSC region, 9 were from the IR region, and there were no fragments from the SSC region. The longest fragment, of $3822 \mathrm{bp}$, originated from the LSC region and included four genes in the chloroplast. The second and third longest migration fragments were about $3646 \mathrm{bp}$ and $3626 \mathrm{bp}$, all from the LSC region. Furthermore, it was found that some small fragment sequences derived from chloroplasts were a subset of large fragment sequences or appeared multiple times in the mitochondrial genome, such as the sequence fragments with the sequence numbers 18 and 19, indicating that these fragments underwent multiple independent migration integrations or that replication and recombination occurred within the mitochondrial genome after migration.

Sequence migration between chloroplast, mitochondrial, and nuclear genomes of watermelon. We analyzed the alignment of sequences between the chloroplast, mitochondrial, and nuclear genomes (Fig. 2). There were 137 sequences in the watermelon chloroplast genome with a total length of about $73 \mathrm{~kb}(47 \%)$ and 567 sequences in the nuclear genome with a total length of about $313 \mathrm{~kb}$. The length of the 


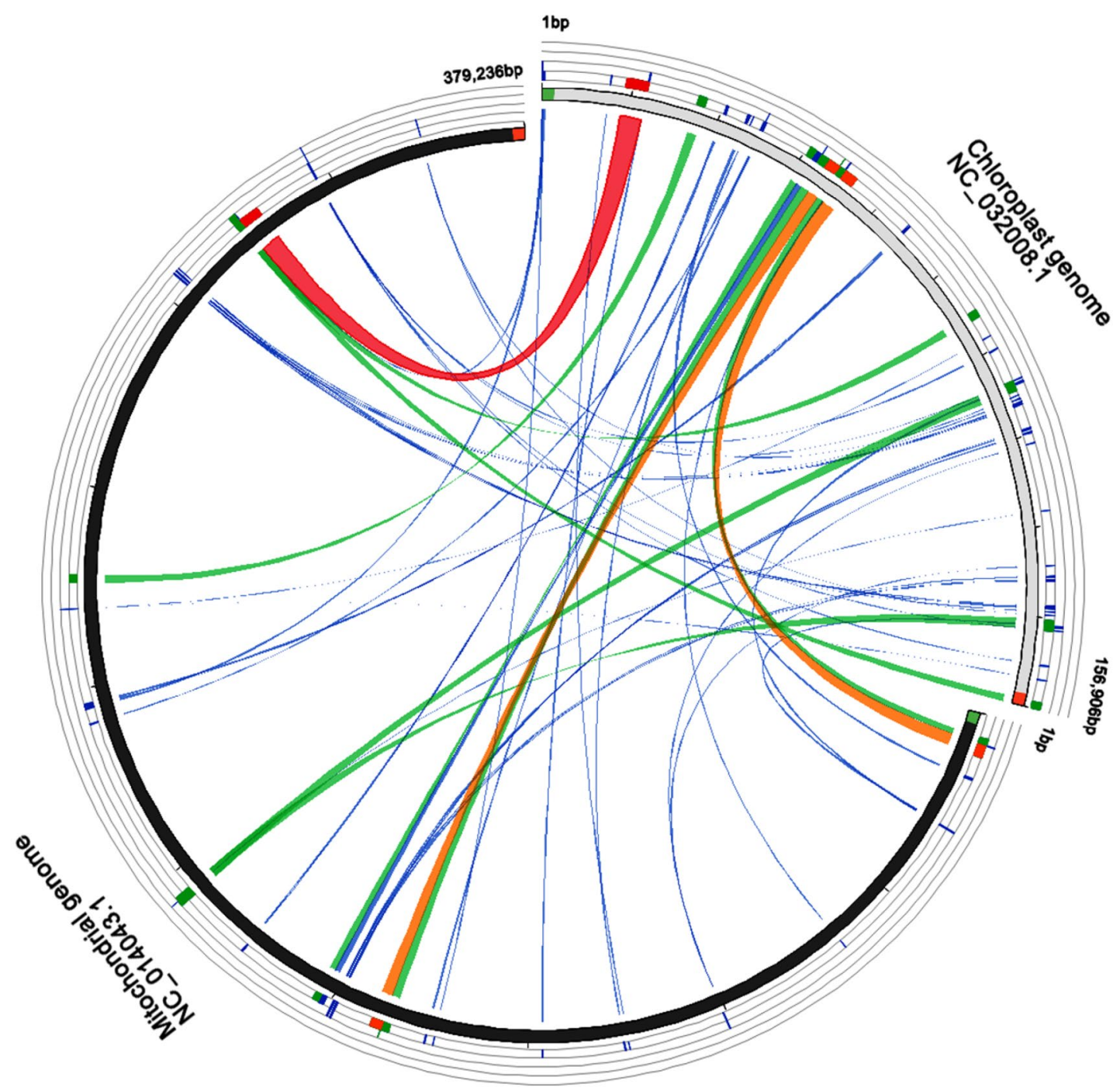

Figure 1. The transfer of sequences from chloroplast to mitochondrial genomes in C. lanatus.

\begin{tabular}{|l|l|l|l|l|}
\hline Fragment length (bp) & Number & Total length (bp) & PTM (\%) & PTC (\%) \\
\hline $50-100$ & 9 & 623 & 0.2 & 0.4 \\
\hline $100-500$ & 12 & 3250 & 0.8 & 2.1 \\
\hline $500-1000$ & 2 & 1726 & 0.5 & 1.1 \\
\hline $1000<$ & 11 & 23,104 & 6.1 & 14.7 \\
\hline Total & 34 & 28,703 & 7.6 & 18.3 \\
\hline
\end{tabular}

Table 3. Summary of the transfer of sequences from chloroplast to mitochondrial genomes in C. lanatus. PTM Percentage of transferred sequences in mitochondrial genome, PTC percentage of transferred sequences in chloroplast genome.

137 sequences in the chloroplast genome ranged from 222 to $4808 \mathrm{bp}$, with an average length of $532 \mathrm{bp}$. The 567 homologous fragments in the nuclear genome were unevenly distributed on 11 watermelon chromosomes. The chloroplast genome had the most homology with chromosome 2 and the least homology with chromosome 5. On average, each homologous chloroplast sequence was similar to four different regions in the nuclear genome. A sequence of about $125 \mathrm{~kb}(33 \%)$ in the watermelon mitochondrial genome was homologous to a $260 \mathrm{~kb}$ sequence in the nuclear genome. The mitochondrial genome had the most sequence homology with chromosome 6, and 263 fragments in the mitochondria corresponded to 520 fragments in the nuclear genome with lengths ranging from 261 to $4246 \mathrm{bp}$ and an average length of $474 \mathrm{bp}$. On average, each mitochondrial fragment was homologous to two different regions on the nuclear genome. These results indicate that, during 

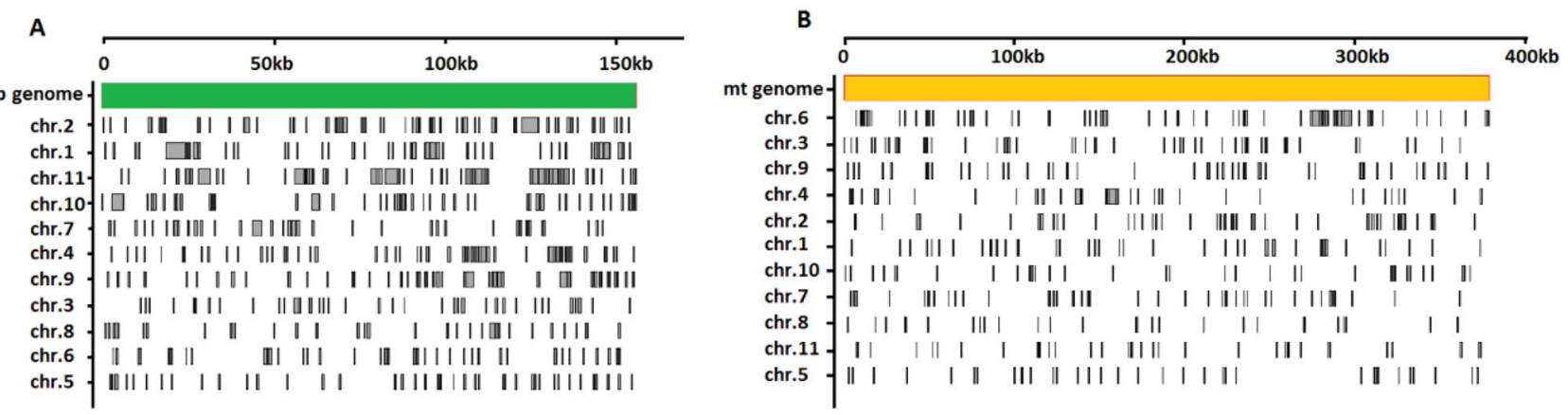

Figure 2. Distribution of homologous genomic sequences between the chloroplast, mitochondria, and nucleus in C. lanatus. (A) Indicates comparisons between chloroplast genome and nuclear genome; (B) indicates comparisons between the mitochondrial and nuclear genomes.

\begin{tabular}{|l|l|l|}
\hline Features & Chloroplast & Mitochondrion \\
\hline Genome size (bp) & 156,017 & $2,906,673$ \\
\hline Chromosome number & 1 & 3 \\
\hline GC (\%) & 36.9 & 44.77 \\
\hline Gene number & 132 & 88 \\
\hline Protein genes number & 87 & 40 \\
\hline Protein genes sequence (bp) & $80,433(51.62 \%)$ & $37,140(1.28 \%)$ \\
\hline Genes with intron (cis/trans) & $18 / 1$ & $10 / 3$ \\
\hline Intron no. (cis/trans) & $24 / 2$ & $17 / 5$ \\
\hline Cis-splicing intron (bp) & $20,286(13.02 \%)$ & $40,886(1.41 \%)$ \\
\hline tRNA number (type) & $37(30)$ & $40(17)$ \\
\hline tRNA sequence (bp) & $2820(1.81 \%)$ & $3284(0.11 \%)$ \\
\hline rRNA number (type) & $8(4)$ & $8(3)$ \\
\hline rRNA sequence (bp) & $10,620(6.81 \%)$ & $5815(0.20)$ \\
\hline
\end{tabular}

Table 4. The basic features of chloroplast and mitochondrial genomes in C. melo. The count excluding the gene duplicates. The note is applicable only to the numbers mentioned in brackets for features tRNA number and rRNA number.

the evolution of watermelon plants, the nuclear and two organelle genomes in the cytoplasm exchanged a large amount of genetic material.

Comparison of chloroplast and mitochondrial genomic characteristics of melon. In the melon, the mitochondrial genome is about 18.7 times the size of the chloroplast genome, but there are less than half the number of protein-coding genes in the mitochondria compared with the chloroplast genome. Furthermore, the mitochondrial protein-coding gene sequences in the melon account for only $1.28 \%$ of the entire mitochondrial genome. In contrast, the chloroplast protein coding gene sequences account for $51.62 \%$ of the entire chloroplast genome (Table 4). The 26 introns of the melon chloroplast are distributed among 18 genes, and a single gene has no more than two introns. The 22 introns of melon mitochondria are distributed among 10 genes. In some genes, such as nad1, nad2, and nad5, there were as many as four introns. Due to frequent recombination of the internal structure of the genome, the number of introns in the melon mitochondrial genome has increased by trans-splicing.

Chloroplast and mitochondrial genomic sequence transfer in melon. In this study, we analyzed the sequences in the melon mitochondrial genome that have migrated from the chloroplast genome and found 91 chloroplast-derived sequences with a length of more than $50 \mathrm{bp}$ and a similarity of no less than $80 \%$ (Fig. 3; Table 5). The total length of migrated sequences was $79,4630 \mathrm{bp}$, about $2.73 \%$ and $50.99 \%$ of the full length of the mitochondrial and chloroplast genomes, respectively. Among the migration sequences, 33 fragments were shorter than $500 \mathrm{bp}, 58$ were longer than $100 \mathrm{bp}, 25$ were longer than $500 \mathrm{bp}$, and 19 fragments were longer than $1 \mathrm{~kb}$ (Supplementary Table S2). Among these fragments, 20 were from the chloroplast genome LSC region, 34 were from the IR regions, and 4 were from the SSC region, indicating that most of the mitochondrial sequences originating from the chloroplast genome came from the repeat regions. The second and third longest migration fragments were approximately $9145 \mathrm{bp}$ and $8968 \mathrm{bp}$, from the LSC and IR regions, respectively. At the same time, it was found that some small fragment sequences derived from chloroplasts were a subset of large fragment sequences or had appeared multiple times in the mitochondrial genome, such as sequence fragments 


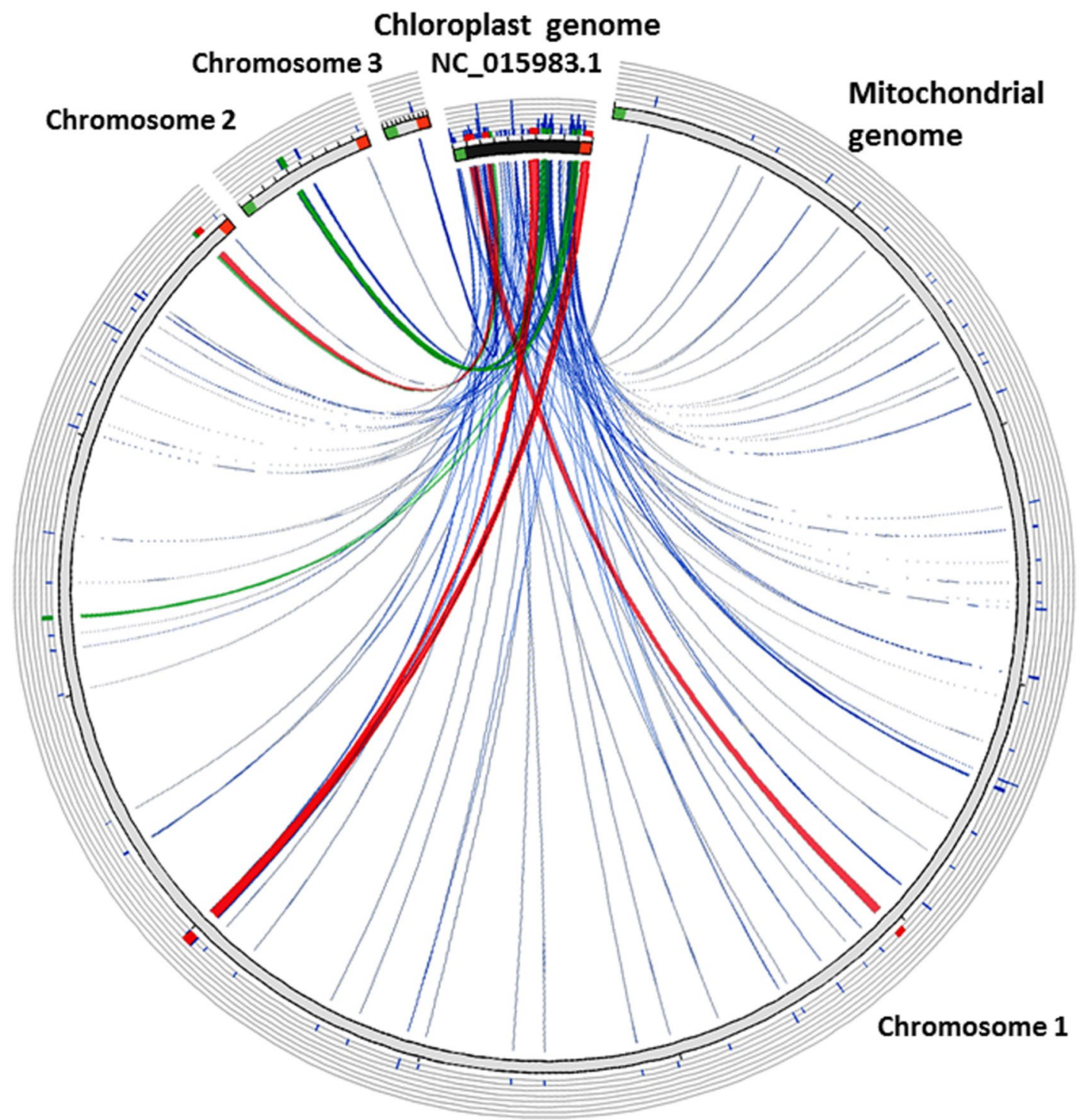

Figure 3. Transfer of sequences from chloroplast to mitochondrial genomes in C. melo.

\begin{tabular}{|l|l|l|l|l|}
\hline Fragment length (bp) & Number & Total length (bp) & PTM (\%) & PTC (\%) \\
\hline $50-100$ & 33 & 1749 & 0.06 & 1.12 \\
\hline $100-500$ & 33 & 6860 & 0.24 & 4.41 \\
\hline $500-1000$ & 6 & 4397 & 0.15 & 2.82 \\
\hline $1000<$ & 19 & 66,457 & 2.28 & 42.65 \\
\hline Total & 91 & 79,463 & 2.73 & 50.99 \\
\hline
\end{tabular}

Table 5. Summary of the transfer of sequences from chloroplast to mitochondrial genomes in C. melo. PTM Percentage of transferred sequences in mitochondrial genome, $P T C$ percentage of transferred sequences in chloroplast genome.

with sequence numbers $34-37$ and 42-45, indicating that these fragments emerged from multiple independent migration and integration events, or replication events, followed by recombination ${ }^{11}$.

Sequence transfer between chloroplast, mitochondrial, and nuclear genomes of melon. Analysis of sequence homologies between the chloroplast, mitochondrial, and nuclear genomes in melon (Fig. 4) showed that there were 108 sequences in the melon chloroplast genome with a total length of approximately $38 \mathrm{~kb}(25 \%)$. In total, 461 sequences with a total length of about $301 \mathrm{~kb}$ showed homologous relationships. The 


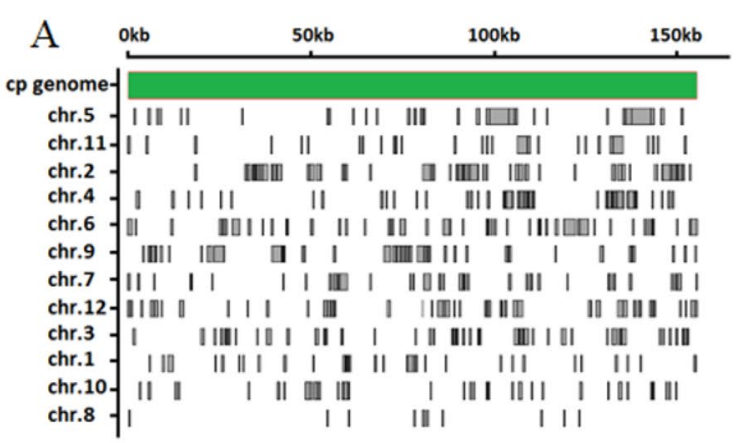

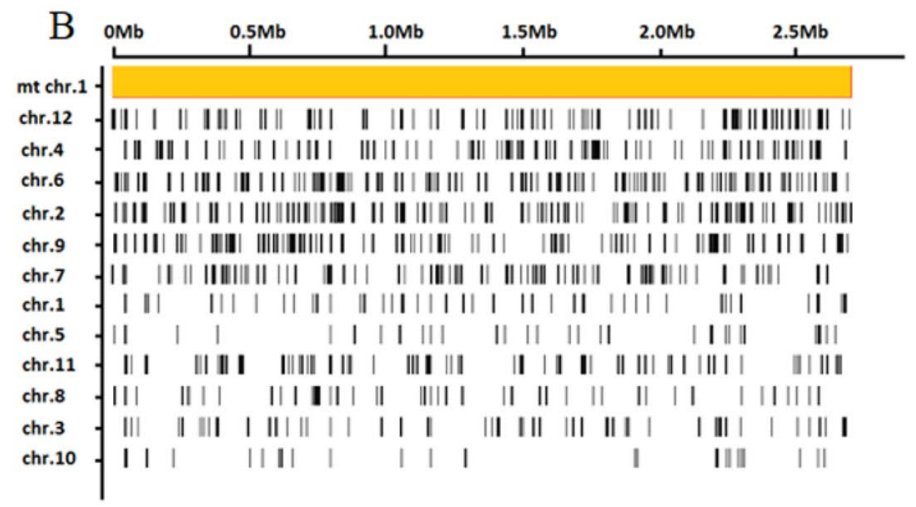

C

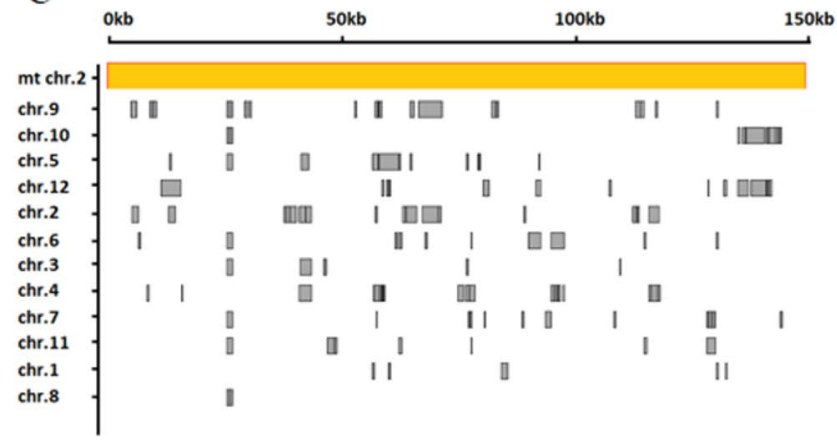

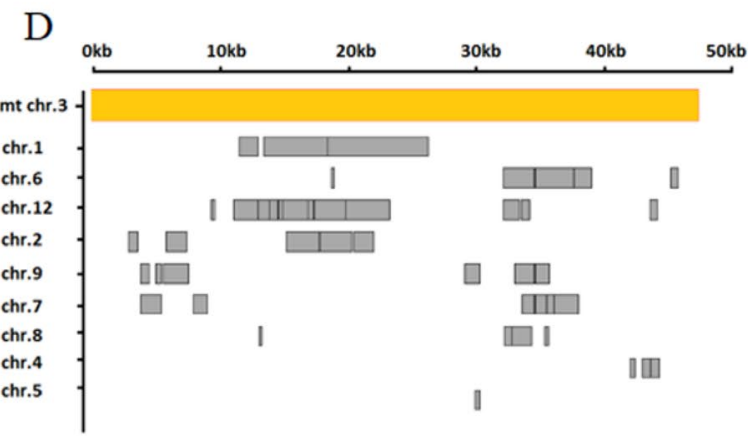

Figure 4. Distribution of homologous sequences within the nuclear, chloroplast, and mitochondrial genomes of C. melo. (A) indicates comparisons between chloroplast and nuclear genomes; (B-D) indicate comparisons between mitochondrial and nuclear genomes.

108 sequences in the chloroplast genome were all $204 \mathrm{bp}$ to $1039 \mathrm{bp}$ in length, with an average length of $363 \mathrm{bp}$. The 461 homologous fragments in the nuclear genome were unevenly distributed throughout the entire melon genome. Among these, chromosome 5 had the most homologous sequences in common with the chloroplast genome, while chromosome 8 had the least homology. On average, each homologous sequence in the chloroplast was similar to four different regions in the nuclear genome; approximately $1413 \mathrm{~kb}(48.62 \%)$ of sequence in the melon mitochondrial genome was homologous to $3.4 \mathrm{Mb}$ of the nuclear genome. While 1048 fragments in the mitochondria corresponded to 3391 fragments on the nuclear genome. On average, each mitochondrial fragment was homologous to three different regions in the nuclear genome. The 1,048 homologous sequences in the mitochondrial genome ranged from $214 \mathrm{bp}$ to $6120 \mathrm{bp}$, with an average length of $941 \mathrm{bp}$.

\section{Discussion}

Sequences that have migrated from chloroplast genomes have been found in the mitochondrial genomes of all plants sequenced so far, and this part of the genome usually accounts for $1-12 \%$ of the total length ${ }^{41}$. It is considered almost impossible for sequences from the plant mitochondrial genome to transfer into the chloroplast genome $^{42}$. The total length of homologous sequences between the melon chloroplast and mitochondrial genomes is approximately $79 \mathrm{~kb}$, about $2.73 \%$ of the whole mitochondrial genome, indicating that these sequences may be derived from the chloroplast. Like that of other angiosperms ${ }^{43}$, the chloroplast genome of the watermelon is relatively conservative and does not accept the integration of foreign DNA fragments. However, the mitochondrial genome contains a large number of sequences that have migrated from the chloroplast genome ${ }^{44}$. The mitochondrial genome of watermelon contains fewer homologous chloroplast genomic sequences, but mitochondria of the zucchini contain $113 \mathrm{~kb}$ of transferred chloroplast genomic sequences, which is approximately $80 \%$ of the total length of the chloroplast genome of the Cucurbitaceae plant ${ }^{11}$. Chloroplast genome fragments usually carry some protein-encoding genes during transfer to the mitochondrial genome, but these genes become non-functional pseudogenes after integration, which may be caused by genomic sequence reorganization ${ }^{45}$. Our analysis of chloroplast migration sequences in the mitochondrial genomes of watermelon and melon supports this conclusion.

Some non-protein-encoding genes, such as tRNA, still perform normal functions after they are transferred to the mitochondrial genome ${ }^{46}$. Compared with the melon, the watermelon's smaller mitochondrial genome contains about $23 \mathrm{~kb}$ of migrated chloroplast sequences, and the zucchini mitochondrial genome contains about $113 \mathrm{~kb}$ of chloroplast sequences, indicating that there is no association between the size of the mitochondrial genome and the amount of chloroplast migration sequences. In this study, we found that the watermelon and melon mitochondrial genomes contain 3 and 17 chloroplast-derived tRNAs with complete structures, respectively, and we believe they may have normal transport functions. This characteristic may be a characteristic of higher plant mitochondria during evolution. At present, there are still many mysteries about the sequence 
migration mechanisms between the organelle genomes in the watermelon and melon and the expression patterns of genes carried by the migrated fragments. However, we believe that further development of the watermelon and melon whole genome project will help in the analysis of these problems.

In addition to the horizontal transfer of gene sequences between chloroplasts and mitochondria, there has also been a rich exchange of genetic material between the two organelle genomes and the nuclear genome. The mitochondrial and nuclear genomes can transfer DNA sequences in both directions. However, most studies have found that the chloroplast genome only transfers DNA sequences to the nuclear and mitochondrial genomes, not to other chloroplasts, and they tend not to integrate foreign $\mathrm{DNA}^{47,48}$. By analyzing the sequence homologies between the chloroplast, mitochondrial, and nuclear genomes, we can estimate the amount of genetic material exchanged between the organelles and the nucleus. Furthermore, the analysis can provide basic data for related research on nuclear-plastid interactions in the watermelon and melon.

\section{Conclusion}

The results indicated that, during the evolution of the watermelon and melon, some small fragments derived from chloroplasts may have undergone multiple independent migrations and integrations, or that replication and recombination occurred after integration, within the mitochondrial genome. This study found that a large amount of genetic material has been exchanged between the genomes. By analyzing the sequence homologies between the chloroplast, mitochondrial, and nuclear genomes, we can estimate the amount of genetic material exchanged between the organelles and the nucleus. Furthermore, the analysis can provide basic data for related research on nuclear-plasmon interactions in the watermelon and melon.

\section{Data availability}

The re-sequencing data of W1-1 and MR-1 were deposited in GenBank (https://www.ncbi.nlm.nih.gov/) under BioProject ID PRJNA682698.

Received: 9 June 2020; Accepted: 17 December 2020

Published online: 15 January 2021

\section{References}

1. Lin, D. A study of systematics for Citrullus Schrad. China Cuc. Veg. 28, 1-4 (2015).

2. Tanaka, K. et al. Diversification and genetic differentiation of cultivated melon inferred from sequence polymorphism in the chloroplast genome. Breed. Sci. 63, 183-196 (2013).

3. Sebastian, P. et al. Cucumber (Cucumis sativus) and melon (C. melo) have numerous wild relatives in Asia and Australia, and the sister species of melon is from Australia. Proc. Natl. Acad. Sci. U. S. A. 107, 14269-14273 (2010).

4. Ali, A. et al. Putative paternal factors controlling chilling tolerance in Korean market-type cucumber (Cucumis sativus L.). Sci. Hortic. Amst. 167, 145-148 (2014).

5. Levi, A. et al. Novel watermelon breeding lines containing chloroplast and mitochondrial genomes derived from the desert species Citrullus colocynthis. HortScience 41, 463-464 (2006).

6. Schaefer, H., Heibl, C. \& Renner, S. S. Gourds afloat: A dated phylogeny reveals an Asian origin of the gourd family (Cucurbitaceae) and numerous oversea dispersal events. Proc. R. Soc. B Biol. Sci. 276, 843-851 (2009).

7. Shinozaki, K. et al. The complete nucleotide sequence of the tobacco chloroplast genome: Its gene organization and expression. EMBO J. 5, 2043-2049 (1986).

8. Swati, T. et al. Comparative analysis of the complete chloroplast genome of mainland Aster spathulifolius and other aster species. Plants Basel 9, 568 (2020).

9. Palmer, J. D. Plastid chromosomes: Structure and evolution. Mol. Biol. Plastids 7, 5-53 (1991).

10. Unseld, M. et al. The mitochondrial genome of Arabidopsis thaliana contains 57 genes in 366,924 nucleotides. Nat. Genet. 15, 57-61 (1997).

11. Alverson, A. J. et al. Insights into the evolution of mitochondrial genome size from complete sequences of Citrullus lanatus and Cucurbita pepo (Cucurbitaceae). Mol. Biol. Evol. 27, 1436-1448 (2010).

12. Notsu, Y. et al. The complete sequence of the rice (Oryza sativa L.) mitochondrial genome: Frequent DNA sequence acquisition and loss during the evolution of flowering plants. Mol. Genet. Genom. 268, 434-445 (2002).

13. Handa, $\mathrm{H}$. The complete nucleotide sequence and RNA editing content of the mitochondrial genome of rapeseed (Brassica napus L.): Comparative analysis of the mitochondrial genomes of rapeseed and Arabidopsis thaliana. Nucleic Acids Res. 31, 5907-5916 (2003).

14. Ogihara, Y., Yamazaki, Y., Murai, K., Kanno, A. \& Tsunewaki, K. Structural dynamics of cereal mitochondrial genomes as revealed by complete nucleotide sequencing of the wheat mitochondrial genome. Nucleic Acids Res. 33, 6235 (2005).

15. Alverson, A. J. et al. Origins and recombination of the bacterial-sized multichromosomal mitochondrial genome of cucumber. Plant Cell 23, 2499-2513 (2011).

16. Li, S. S. et al. Progress on sequencing and alignment analysis of higher plant mitochondrial genomes. J. China Agric. Univ. 16, 22-27 (2011).

17. Gao, C. et al. Horizontal gene transfer in plants. Funct. Integr. Genom. 14, $23-29$ (2014).

18. Goremykin, V. V. et al. The mitochondrial genome of Malus domestica and the import-driven hypothesis of mitochondrial genome expansion in seed plants. Plant J. 71, 615-626 (2012).

19. Sloan, D. B. \& Wu, Z. History of plastid DNA insertions reveals weak deletion and at mutation biases in angiosperm mitochondrial genomes. Genome Biol. Evol. 6, 3210-3221 (2014).

20. Gandini, C. L. \& Sanchez-Puerta, M. V. Foreign plastid sequences in plant mitochondria are frequently acquired via mitochondrionto-mitochondrion horizontal transfer. Sci. Rep. 7, 43402 (2017).

21. Wang, X. C. et al. Diversity of mitochondrial plastid DNAs (MTPTs) in seed plants. Mitochondrial DNA A 4, 635-642 (2017)

22. Smith, D. R. Mitochondrion-to-plastid DNA transfer: It happens. New Phytol. 202, 736-738 (2014).

23. Downie, S. R. \& Jansen, R. K. A comparative analysis of whole plastid genomes from the Apiales: Expan-sion and contraction of the inverted repeat, mitochondrial to plastid transfer of DNA, and identification of highly divergent noncoding regions. Syst. Bot. 40, 336-351 (2015).

24. Ma, P. F. et al. Evidence for horizontal transfer of mitochondrial DNA to the plastid genome in a bamboo genus. Sci. Rep. 5, 11608 (2015). 
25. Timmis, J. N., Ayliffe, M. A., Huang, C. Y. \& Martin, W. Endosymbiotic gene transfer: Organelle genomes forge eukaryotic chromosomes. Nat. Rev. Genet. 5, 123-135 (2004).

26. Stern, D. B. \& Lonsdale, D. M. Mitochondrial and chloroplast genomes of maize have a 12 - kilobase DNA-Sequence in common. Nature 299, 698-702 (1982).

27. Schuster, W. \& Brennicke, A. Plastid, nuclear and reverse-transcriptase sequences in the mitochondrial genome of Oenothera: Is genetic information transferred between organelles via RNA?. EMBO J. 6, 2857-2863 (1987).

28. Grewe, F., Viehoever, P., Weisshaar, B. \& Knoop, V. A trans-splicing group I intron and tRNA-hyperediting in the mitochondrial genome of the lycophyte Isoetes engelmannii. Nucleic Acids Res. 37, 5093-5104 (2009).

29. Rice, D. W. et al. Horizontal transfer of entire genomes via mitochondrial fusion in the angiosperm Amborella. Science 342, 1468-1473 (2013).

30. Allen, G. C. et al. A modified protocol for rapid DNA isolation from plant tissues using cetyltrime thylammonium bromide. Nat. Protoc. 1, 2320-2325 (2006).

31. Guo, S. et al. The draft genome of watermelon (Citrullus lanatus) and resequencing of 20 diverse accessions. Nat. Genet. 45, 51-58 (2013).

32. Garcia-Mas, J. et al. The genome of melon (Cucumis melo L.). Proc. Natl. Acad. Sci. U. S. A. 109, 11872-11877 (2012).

33. Takagi, H. et al. QTL-seq: Rapid mapping of quantitative trait loci in rice by whole genome resequencing of DNA from two bulked populations. Plant J. 74, 174-183 (2013).

34. Li, H. Aligning sequence reads, clone sequences and assembly contigs with BWA-MEM. ArXiv Preprint ArXiv 1303, 3997 (2013).

35. Frith, M. C., Hamada, M. \& Horton, P. Parameters for accurate genome alignment. BMC Bioinform. 11, 80 (2010).

36. Chen, Y. et al. High speed BLASTN: An accelerated MegaBLAST search tool. Nucleic Acids Res. 43, 7762 (2015).

37. Chen, C. et al. TBtools: An integrative toolkit developed for interactive analyses of big biological data. Mol. Plant 13, 1194-1202 (2020).

38. Ihaka, R. \& Gentleman, R. R: A language for data analysis and graphics. J. Comput. Graph. Stat. 5, 299-314 (1996).

39. Naquin, D., d'Aubenton-Carafa, Y., Thermes, C., at el. CIRCUS: A package for Circos display of structural genome variations from paired-end and mate-pair sequencing data. BMC Bioinform. 15, 198 (2014).

40. Ginestet, C. ggplot2: Elegant graphics for data analysis. J. R. Stat. Soc. A Stat. 174, 245 (2011)

41. Mower, J. P., Sloan, D. B., Alverson, A. J. Plant mitochondrial genome diversity: The genomics revolution. In: Plant Genome Diversity, Vol. 1 (eds Wendel, J., Greilhuber, J., Dolezel, J., Leitch, I.) 123-144 (Springer, Vienna, 2012).

42. Leister, D. Origin, evolution and genetic effects of nuclear insertions of organelle DNA. Trends Genet. 21, 655-663 (2005).

43. Katayama, H. et al. Phylogenetic utility of structural alterations found in the chloroplast genome of pear: Hypervariable regions in a highly conserved genome. Tree Genet. Genomes 8, 313-326 (2012).

44. Nakazono, M. \& Hirai, A. Identification of the entire set of transferred chloroplast DNA sequences in the mitochondrial genome of rice. Mol. Gen. Genet. 236, 341-346 (1993).

45. Richardson, A. O. et al. The "fossilized" mitochondrial genome of Liriodendron tulipifera: Ancestral gene content and order, ancestral editing sites, and extraordinarily low mutation rate. BMC Biol. 11, 29 (2013).

46. Kitazaki, K. et al. A horizontally transferred tRNACys gene in the sugar beet mitochondrial genome: Evidence that the gene is present in diverse angiosperms and its transcript is aminoacylated. Plant J. Cell Mol. Biol. 68, 262-272 (2011).

47. Huang, C. Y. Mutational decay and age of chloroplast and mitochondrial genomes transferred recently to angiosperm nuclear chromosomes. Plant Physiol. 138, 1723-1733 (2005).

48. Han, A. D., Wang, C. X., Xiao, Z., et al. pRRD9, a repetitive DNA sequence that transfers from the chloroplast genome to the nuclear genome with diverse amplification across species. Rice Genet. III 1028(1996).

\title{
Acknowledgements
}

This study was funded by the National Nature Science Foundation of China (No. 31672177).

\section{Author contributions}

Conceptualization, Q.Z.; data curation, Y.W. and B.Q.; funding acquisition, P.G.; writing_original draft, Z.D.; writing-review and editing, H.C.

\section{Competing interests}

The authors declare no competing interests.

\section{Additional information}

Supplementary Information The online version contains supplementary material available at https://doi. org/10.1038/s41598-020-80149-9.

Correspondence and requests for materials should be addressed to P.G.

Reprints and permissions information is available at www.nature.com/reprints.

Publisher's note Springer Nature remains neutral with regard to jurisdictional claims in published maps and institutional affiliations.

\begin{abstract}
(c) (1) Open Access This article is licensed under a Creative Commons Attribution 4.0 International cc. License, which permits use, sharing, adaptation, distribution and reproduction in any medium or format, as long as you give appropriate credit to the original author(s) and the source, provide a link to the Creative Commons licence, and indicate if changes were made. The images or other third party material in this article are included in the article's Creative Commons licence, unless indicated otherwise in a credit line to the material. If material is not included in the article's Creative Commons licence and your intended use is not permitted by statutory regulation or exceeds the permitted use, you will need to obtain permission directly from the copyright holder. To view a copy of this licence, visit http://creativecommons.org/licenses/by/4.0/.
\end{abstract}

(C) The Author(s) 2021 